\title{
Use of inverse stability solutions for identification of uncertainties in the dynamics of machining processes
}

\author{
Lutfi Taner Tunc ${ }^{1}\left(\mathbb{D} \cdot\right.$ Orkun Ozsahin $^{2}$
}

Received: 24 October 2017/ Accepted: 13 July 2018

(C) Shanghai University and Springer-Verlag GmbH Germany, part of Springer Nature 2018

\begin{abstract}
Research on dynamics and stability of machining operations has attracted considerable attention. Currently, most studies focus on the forward solution of dynamics and stability in which material properties and the frequency response function at the tool tip are known to predict stable cutting conditions. However, the forward solution may fail to perform accurately in cases wherein the aforementioned information is partially known or varies based on the process conditions, or could involve several uncertainties in the dynamics. Under these circumstances, inverse stability solutions are immensely useful to identify the amount of variation in the effective damping or stiffness acting on the machining system. In this paper, the inverse stability solutions and their use for such purposes are discussed through relevant examples and case studies. Specific areas include identification of process damping at low cutting speeds and variations in spindle dynamics at high rotational speeds.
\end{abstract}

Keywords Inverse stability $\cdot$ Machining dynamics $\cdot$ High speed milling $\cdot$ Process damping $\cdot$ Spindle dynamics

Lutfi Taner Tunc

ttunc@sabanciuniv.edu

1 Integrated Manufacturing Technologies Research and Application Center, Faculty of Engineering and Natural Sciences, Sabanci University, 34956 Tuzla, Istanbul, Turkey

2 Department of Mechanical Engineering, Middle East Technical University, Ankara, Turkey

\section{Introduction}

The analysis and simulation of machining dynamics and stability constitutes one of the mostly widely examined topics in machining research since the first cutting tests by Taylor [1]. In machining, chatter is a major limitation that is handled and avoided by identifying stable cutting conditions. Stability analysis is the most widely used analytical tool in extant studies $[2,3]$ in which the prediction of stable cutting conditions leads to significant improvements in machining performance and part quality. Most previous studies rely on the forward solution of the stability problem in which the frequency response function (FRF) at the tool tip and the material properties are well known.

The first effort to predict stable cutting conditions commenced with studies on the simplest case of orthogonal cutting by Tlusty and Polacek [4], and Das and Tobias [5]. In an early study on modeling dynamics and stability of flat end milling, Koenigsberger and Tlusty [6] utilized the orthogonal cutting stability model on milling by defining an average cut direction and average number of teeth in the cut. Although this was a rough estimate for general milling conditions, it provided a better understanding of a solution for milling stability. Opitz and Bernardi [7] subsequently improved on the approach by introducing a varying directional coefficient that accounted for the sinusoidal variation in the oriented FRFs along the chip thickness and tangential directions. The first attempt to predict stability limits in end milling was presented by Sridhar et al. [8]. It provided details on the fundamentals of the modern milling stability model in the frequency domain in which they included the effect of different harmonics of the dynamic milling forces [8]. They used the correct orientation of cutting forces as opposed to applying an average direction. In order to accurately predict the stability limits in end 
milling, Floquet's theorem and Nyquist stability criterion were used by Minis et al. [9], and this was followed by the first analytical solution to the milling stability problem by Altintas and Budak [2]. With respect to complicated end milling cases in which irregular cutting edges were utilized, Insperger and Stepan [3] proposed the successful application of the semi-discretization method.

Process damping phenomenon is mostly emphasized as the most common reason for variation and uncertainties in machining dynamics at low cutting speeds [10]. In early studies, the process damping forces were modeled as a function of the dynamic cutting force coefficients [10], and this led to inconsistent data and analysis. Subsequently, it was related to the indentation between the tool flank face and the workpiece undulations were indicated as the main source of process damping [11, 12]. This approach led to more consistent modeling efforts. Nevertheless, the dynamics and stability problem is handled by using the forward solution in most extant studies. The first inverse stability solution for modeling the process damping was proposed by Budak and Tunc [13], and the average process damping coefficients were calculated in terms of experimental stability limits.

Generally, tool tip FRF is measured at the idle state of the spindle. This may lead to inaccuracies to predict chatter stability limits given variations in spindle dynamics and especially if elements with high inertia rotate at high spindle speeds. Under such operational conditions, bearing stiffness and damping may change due to gyroscopic moments, centrifugal forces, and thermal expansions [14-16]. Variations in bearing parameters result in deviations in the tool point FRF, and thereby in the stability of machining operations. Additionally, spindle shaft, holder, and tool dynamics may change due to the centrifugal forces and gyroscopic moments, and each mode may separate into backward and forward modes [17, 18]. Furthermore, the drawbar mechanism is also affected by high rotational speed conditions [19], and the drawbar force decreases due to the centrifugal force leading to decreases in the contact stiffness at the spindle-holder interface. Therefore, it is necessary to consider the variations in the dynamics of each component in modeling to accurately predict in-process FRFs. In an early study, Kruth et al. [20] proposed an inverse stability solution to identify the FRF (especially at high frequency components) and obtain stability lobes at high speed milling conditions without measuring FRF. In addition to this identification method, they proposed an approach to select optimal cutting parameters for chatterfree material removal. Kilic et al. [21] used an inverse stability solution approach to extract the modal parameters of machine tools that could vary due to thermal issues and rotational affects. They focused on investigating the effects of tool wear on milling stability. They considered several cases including the single mode and symmetrical modes. Subsequently, Suzuki et al. [22] extended the approach to miniature milling tools with small diameters, such as $6 \mathrm{~mm}$, in which the direct measurement of tool tip dynamics through impact hammer tests might not be practically feasible. They used chatter tests to identify the tool tip dynamics in these cases.

Finite element (FE) is the most commonly used method for the modeling of spindle units. In FE models, the spindle shaft, tool holder, and tool are modeled by using Timoshenko beam elements and coupled with the nonlinear bearing models in which the effects of centrifugal forces and gyroscopic moment are included. Although several spindle-bearing models are proposed in extant studies [19, 23-25], accurate prediction continues to constitute a challenge, and may not be possible in a few cases. One of the main reasons for this is that several machine tool users do not possess necessary information such as the spindle geometry and bearing preload amounts and their variations with speed. Furthermore, the bearing dynamics can change at some time during the operation.

Due to the limitations in modeling approaches, there are several experimental studies that focus on identifying the aforementioned variations leading to uncertainties in machine tool dynamics under operational conditions [26-32]. A similarity of these experimental studies is that they require complicated experimental setups and the solution of signal processing problems. However, all these limitations are eliminated in a recent study proposed by Ozsahin et al. [33] in which chatter tests are directly used for the identification of tool point FRF under operational conditions. They implemented the inverse stability solution method to identify tool point FRF and showed that tool point FRF under operational conditions could be accurately identified without complicated experimental setups.

Following a summary of the efforts on modeling and simulation of dynamics and stability of machining operations, the sources for uncertainties are also indicated. The major factors leading to uncertainty in machining dynamics correspond to process damping, and varying spindle dynamics, which may require either complicated test setups or complicated process models in forward stability solution approaches. However, the use of inverse stability solutions may lead to significant simplifications in both experimental and modeling efforts. In this study, previously proposed inverse stability approaches are summarized in a comprehensive manner for this purpose. Although, the study does not provide any new experimental results, the aim of the study is to provide an understanding on the use of an inverse stability solution in dynamics and stability analysis. Henceforth, the study is organized as follows. The dynamics and stability of turning and milling processes are summarized in the next section. This is continued with the 
use of an inverse stability solution in a low cutting speed region. Subsequently, the inverse stability solution is used to identify uncertainties in high rotational speed regions due to the variations in spindle dynamics. The conclusions are then discussed.

\section{Dynamics and stability of machining operations}

The dynamics and stability of machining operations are mostly examined with respect to two main groups, namely turning and milling in which the fundamental difference involves the periodicity of the cutting process. In this section, the dynamics and stability of the aforementioned machining processes are briefly discussed to emphasize the use and importance of inverse stability solutions to deal with uncertainties.

\subsection{Orthogonal turning}

A single degree of freedom (SDOF) orthogonal cutting system is represented in Fig. 1 in which the modal stiffness $k$, structural damping $c$, and modal mass $m$, are depicted in conjunction with basic cutting parameters such as the cutting speed $V$ and feed rate.

The equation of motion for the SDOF orthogonal cutting system is expressed in terms of the modal parameters as

$\left\{\begin{array}{l}m \ddot{x}(t)+c \dot{x}(t)+k x(t)=F_{x}(t), \\ F_{x}(t)=K_{\mathrm{f}} b\left(h_{0}-x(t)+x(t-\tau)\right),\end{array}\right.$

where $h_{0}$ is the static chip thickness, $x(t)$ the instantaneous displacement, $F_{x}(t)$ the instantaneous cutting force in chip thickness direction. In orthogonal cutting, low spindle speeds are typically utilized, and thus the absolute stability limit is of primary interest. Following mathematical manipulations and converting the equation of motion from time domain to the frequency domain, the absolute stability limit $a_{\text {lim }}$, of the orthogonal cutting process is derived in terms of the cutting force coefficient $K_{\mathrm{f}}$, and the minimum

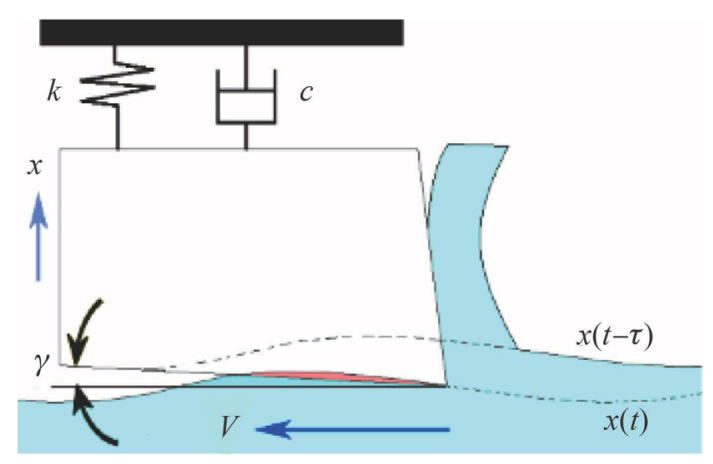

Fig. 1 SDOF orthogonal cutting and flank-wave contact [13] of the real part of the complex frequency response function $\operatorname{Re}(G)_{\min }$, as follows

$a_{\mathrm{lim}}=-\frac{1}{2 K_{\mathrm{f}} \operatorname{Re}(G)_{\min }}$.

After re-writing the real part of the frequency response function in Eq. (2) in terms of the modal parameters, the absolute stability limit is approximated for low damping systems, i.e., $\zeta<10 \%$, and is expressed as

$a_{\lim } \approx \frac{2 k \zeta}{K_{\mathrm{f}}}$.

In Eq. (3), the absolute stability evidently changes when either the cutting force coefficient or the modal parameters change. The variation in the cutting force coefficient may depend on the cutting speed or the feed rate based on the cutting conditions, and this may be calibrated. It may not be straightforward to predict and account for any variation in the stiffness or the damping ratio. Furthermore, given the uncertainty or lack of knowledge of the aforementioned parameters, the accurate prediction of the absolute stability limit is almost impossible.

Extant studies indicate that the variation of stiffness is associated with the variation in the bearing stiffness [15] while the variation in the damping coefficient is related to the process damping [10] that arises at low cutting speeds. Nevertheless, the quantification of these variations is immensely important in several studies that may require the inverse solution of the stability equation as discussed in Sect. 4 .

\subsection{End milling}

In milling, both the absolute stability limit and the stability pockets are of interest for improved process productivity given the interrupted cutting nature of the process and since the diameter of the rotating counterpart, i.e., cutting tool, is significantly lower. A cross sectional view of a helical end mill with flexibility in the $x$ and $y$ directions is shown in Fig. 2.

The corresponding equation of motion in the time domain for this type of a 2-DOF system is expressed in terms of the modal parameters and the instantaneous cutting forces in two directions as

$m_{u} \ddot{u}+c_{u} \dot{u}+k_{u} u=F_{u}(t), \quad u=x, y$,

where $k_{u}$ is the modal stiffness, $c_{u}$ the modal damping coefficient, $m_{u}$ the modal mass, $\dot{u}$ the vibration velocity, $\ddot{u}$ the vibration acceleration and $F_{u}(t)$ the dynamic cutting force along direction $u$. In Eq. (4), the equations of motion are shown as decoupled in $x$ and $y$ directions although they are coupled along the chip thickness and tangential directions [2]. Subsequently, they are included in the frequency domain solution in the form of directional coefficients. 


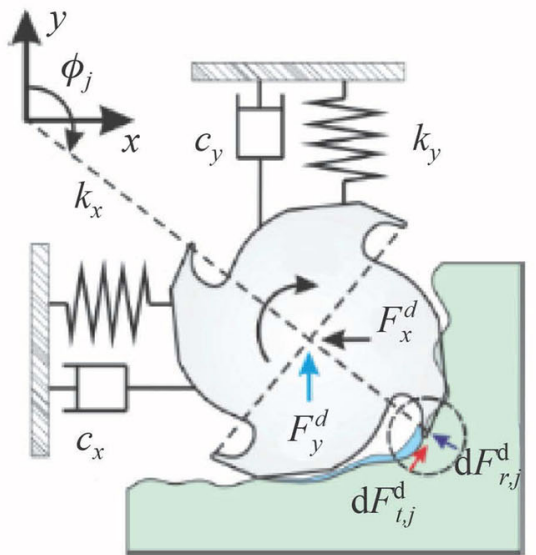

(a)

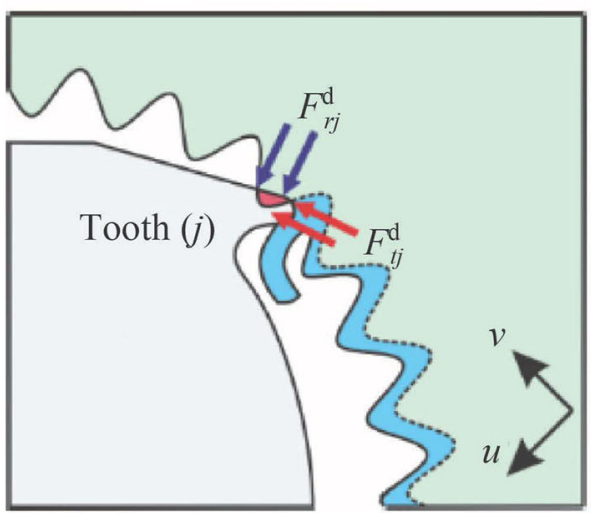

(b)

Fig. 2 Cross section of a helical end mill [34] a end milling system, b flank-workpiece interaction

In a manner similar to turning stability, after mathematical manipulations on the equation of motion, the stability limit is analytically derived in the frequency domain and in terms of the complex eigenvalue of the system, $\Lambda$, tangential cutting force coefficient $K_{\mathrm{t}}$, and number of cutting edges $N$ [2]

$a_{\lim }=-\frac{2 \pi \Lambda_{\mathrm{R}}}{N K_{t}}\left(1+k^{2}\right)$,

where $\Lambda=-\frac{1}{2 a_{0}}\left(a_{1} \pm \sqrt{a_{1}^{2}-4 a_{0}}\right), k=\frac{\Lambda_{\mathrm{I}}}{\Lambda_{\mathrm{R}}}, a_{0}=G_{x x} G_{y y}$ $\left(\alpha_{x x} \alpha_{y y}-\alpha_{x y} \alpha_{y x}\right), a_{1}=\alpha_{x x} G_{x x}+\alpha_{y y} G_{y y}$.

In Eq. (5), $\Lambda_{\mathrm{R}}$ denotes the real part of the complex eigenvalue $\Lambda$, and $a_{0}$ and $a_{1}$ are expressed in terms of the directional coefficients and the FRF [2]. The complex eigenvalue is a function of the frequency response function, and the stability limit at a chatter frequency, $\omega_{\mathrm{c}}$, is a function of the frequency response function and thereby the modal parameters. Therefore, uncertainty in the cutting force coefficients or in the modal parameters leads to variations in stability limits.
$G_{u v}^{t}\left(\mathrm{i} \omega_{c}\right)=\frac{1-\rho_{u v}^{2}+2 \mathrm{i} \zeta_{u v}^{t} \rho_{u v}^{2}}{k_{u v}\left(\left(1-\rho_{u v}^{2}\right)^{2}+\left(2 \zeta_{u v}^{t} \rho_{u v}\right)^{2}\right)}$,

where $\rho_{u v}=\frac{\omega_{\mathrm{c}}}{\omega_{n, u v}}, \quad u=x, y, \quad v=x, y$.

Thus, at a given spindle speed, when the stability limit and the corresponding chatter frequency are known, the unknown parameter can be extracted from the inverse solution of the stability formulation. Furthermore, in the case in which the FRF is unknown, it is identified through the best fitting of the simultaneous solution of the stability equations expressed by using the multiple spindle speed and corresponding stability limit pairs. The rest of the study details aforementioned approaches with relevant examples.

\section{Inverse stability solution at low cutting speeds}

In dynamic cutting, undulations develop on the machined surface due to vibrations. At low cutting speed regions, the waves become steeper when the cutting speed decreases and lead to interference between the tool flank face and the undulation left on the workpiece (see Fig. 3a). Thus, this leads to an additional damping force that is termed as the process damping in extant studies on machining. This phenomenon is valid for both turning and milling operations given the same fundamental understanding albeit in a different geometrical fashion. It is crucial to identify the

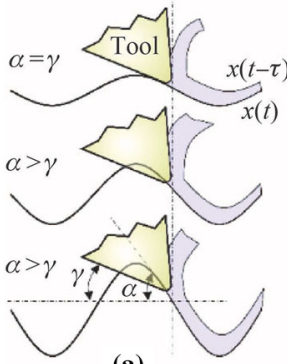

(a)

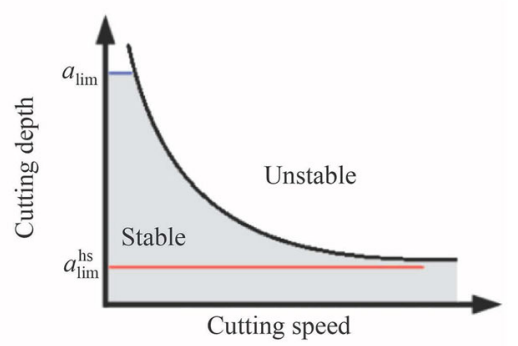

(b)

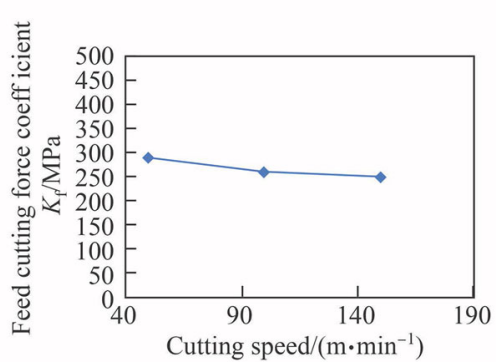

(c)

Fig. 3 Effect of the cutting speed process mechanics and dynamics a tool-workpiece indentation, b absolute stability versus cutting speed, and $\mathbf{c}$ cutting force coefficient versus cutting speed 
amount of the additional process damping acting on the machining system for modeling purposes, and this can then be used to predict the amount of process damping by relating the process damping coefficient to the tool: workpiece indentation volume. In this section, the use of inverse stability solutions is discussed by examining the theory behind it and representative examples.

\subsection{Inverse stability solution in turning operations}

In orthogonal cutting, the minimum of the real part of the FRF can be approximated in terms of the modal parameters [13], the absolute stability limit is derived in terms of the modal parameters as given in Eq. (3). It is assumed that $k$ and $K_{\mathrm{f}}$ remain almost constant (see Fig. 3c) with decreases in cutting speed, and thus it is known that the main source of stability increase corresponds to the increase in the damping ratio. Hence, the difference between the absolute stability limits at high cutting speeds, i.e., $a_{\text {lim }}^{\text {hi }}$, and low cutting speeds (i.e., $\left.a_{\mathrm{lim}}^{\mathrm{low}}\right)$ can be used to identify the difference between the total damping ratio and the structural damping ratio, thereby leading to the process damping ratio.

After re-writing Eq. (3), the total process damping coefficient $c_{\mathrm{t}}$, is expressed as given below in which the cutting force coefficient and natural frequency $\omega_{\mathrm{n}}$, do not change with respect to the cutting speed.

$c_{\mathrm{t}}=\frac{K_{\mathrm{f}} a_{\mathrm{lim}}^{\text {low }}}{\omega_{\mathrm{n}}}$,

where $\omega_{\mathrm{n}}=\sqrt{\frac{k}{m}}$.

The inverse stability solution is given in Eq. (7) of the orthogonal turning stability and provides an excellent method to quantify the average process damping coefficient by subtracting the structural damping from the total damping ratio as

$c_{\mathrm{p}}=\frac{K_{\mathrm{f}} a_{\mathrm{lim}}^{\text {low }}}{\omega_{\mathrm{n}}}-c_{\mathrm{s}}$.

After a simple mathematical manipulation, $K_{\mathrm{f}}$ is expressed in terms of the structural damping. Thus, the process damping coefficient is simplified as given in Eq. (9). This provides an extremely practical inverse solution to calculate the amount of average process damping coefficient acting on the system when the structural damping, absolute stability limit at the corresponding low cutting speed, and absolute stability limit at a relatively high cutting speed are known

$c_{\mathrm{p}}=c_{\mathrm{s}}\left(\frac{a_{\mathrm{lim}}^{\mathrm{low}}}{a_{\mathrm{lim}}^{\mathrm{hi}}}-1\right)$.

The experimental process damping coefficients obtained in Eq. (9) are further used for the modeling of process damping coefficients to simulate absolute stability limits under various other cutting conditions, and this eliminates the need to test each case. This is performed by introducing the indentation coefficient that is extracted by the damping energy analysis as detailed in Ref. [13].

\subsection{Inverse stability solution in milling operations}

In end milling, given the two degrees of freedom and directional interaction, the stability limit cannot be expressed by a simple equation involving the modal parameters and cutting force coefficient as in orthogonal cutting. However, the inverse stability solution approach can be still used in end milling when the spindle speed, stability limit at the low cutting speed, chatter frequency, and stability limit at high cutting speeds (where process damping is negligible) are known. Given that the stability lobes can be realized in milling, in the experimental phase, it is important to select the spindle speeds corresponding to the same chatter frequency at consecutively increasing lobe numbers. Hence, each spindle speed and stability limit pair can be analyzed at the same chatter frequency, thereby leading to increases in comparable ratios of the stability limit.

With respect to the identification of process damping coefficients, the inverse solution of the analytical stability expression [2] given in Eq. (5) is used. It is solved iteratively by considering the implicit form of the stability expression given in Eq. (5) [34]. It is assumed that average process damping coefficients act on the end milling system in two orthogonal directions due to the instantaneous flankwave indentation, and thus the overall FRFs are expressed by including the effect of the virtual damping pots due to the process Eq. (6). When the experimental stability limit, $a_{\text {lim }}^{\text {low }}$, at the corresponding low cutting speed and the chatter frequency $\omega_{\mathrm{c}}$, are known, the experimental eigenvalue $\Lambda_{\text {exp }}$, is calculated by using the experimental values by rewriting Eq. (5) as given below. Subsequently, it is equated to the analytical eigenvalue that relates the overall FRF to the stability limit

$$
\left\{\begin{array}{c}
\Lambda_{\exp }=\Lambda_{\text {analytical }}, \\
\frac{a_{\lim } N K_{\mathrm{t}}\left(1-\cos \omega_{\mathrm{c}} T\right)}{4 \pi}-\frac{a_{\lim } N K_{\mathrm{t}} \sin \omega_{\mathrm{c}} T}{4 \pi}=-\frac{1}{2 a_{0}}\left(a_{1} \pm \sqrt{a_{1}^{2}-4 a_{0}}\right) .
\end{array}\right.
$$

The solution of the nonlinear Eq. (10) leads to the total damping ratios in the $x$ and $y$ directions acting on the system. However, an explicit solution for this equality is not available, and thus it is solved through an iterative solution based on the golden section search algorithm. Finally, the process damping coefficients are identified as

$c_{i}^{\mathrm{p}}=2\left(\zeta_{i}^{\mathrm{t}}-\zeta_{i}^{\mathrm{s}}\right) \sqrt{k_{i} m_{i}}, \quad i=x, y$, 
where $c_{i}^{\mathrm{p}}, \zeta_{i}^{\mathrm{t}}$ and $\zeta_{i}^{\mathrm{s}}$ denote the process damping coefficient, total damping ratio, and structural damping ratio, along direction $i$, respectively.

The analytical identification procedure relies on the inverse stability solution of the average process damping coefficients in orthogonal turning and end milling operations and is summarized based on extant studies in this section. In turning, the main interest involves the absolute stability limits since the stability lobes cannot be realized at lower spindle speed regions. An explicit analytical approximation of the absolute stability limit in terms of the modal parameters is used to express the process damping coefficients in terms of the structural damping and ratio of the stability limit at low cutting speed to that obtained in the high cutting speed region. However, in milling, the explicit analytical expression for the stability limit in terms of the modal parameters is not available due to the existence of the two orthogonal modes. In these cases, the inverse stability equation is solved through iteration, and the process damping coefficients are calculated in the $x$ and $y$ directions. Henceforth, this section demonstrates the use of the summarized procedures in case studies.

\subsubsection{Orthogonal cutting}

The inverse stability solution in orthogonal cutting is demonstrated with respect to the turning of a $100-\mathrm{mm}$ diameter tube composed of AL7075 in dry conditions with triangular carbide inserts involving a hone radius of $60 \mu \mathrm{m}$ and a clearance angle of $3^{\circ}$ at the flank face as given in Table 1. Absolute stability limits are experimentally identified at decreasing cutting speeds by analyzing the sound, tool vibration, and the resulting surface. Subsequently, the absolute stability limits are used in Eq. (9) to calculate the average process damping coefficients. The details of the test conditions are given in Ref. [13].

Figure $4 \mathrm{a}$ shows the variation in the absolute stability limit with the cutting speed and corresponding process damping coefficients. In Test 1 , the stability limit at relatively high speed of $150 \mathrm{~m} / \mathrm{min}$ corresponds to $3 \mathrm{~mm}$, and this tends to increase nonlinearly when the cutting speed is decreased to $75 \mathrm{~m} / \mathrm{min}$. The increase in the stability limit is observed for a lower cutting speed region in Test 2. This is due to the low natural frequency of the system used in Test 2. At low vibration frequencies, the undulations on the workpiece surface become smoother, thereby leading to less tool-workpiece indentation.

The process damping coefficients as calculated by using Eq. (9) are plotted in Fig. 4b in which the variation in the process damping coefficient is significantly nonlinear. The calculated process damping coefficients can be used for a curve fit to predict the stability limits at other cutting speeds while ensuring that the cutting tool and workpiece are identical. The use of inverse stability solution is demonstrated for orthogonal cutting through two experimental cases, and this is further extended to end milling in the next section.

\subsubsection{End milling}

The end milling case is illustrated for the conditions and milling system listed in Table 2. The milling system demonstrates almost symmetrical dynamic properties in the two directions.

Figure 5 shows a comparison of the analytical stability diagrams calculated for the system (by ignoring the process damping effect) and experimental absolute stability limits. In Fig. 5, the dots and continuous curve represent the experimental data and the analytical stability lobes, respectively. In the design of experiments to determine the experimental spindle speeds, it is necessary to eliminate the lobing effect to clearly identify the process damping coefficients. Hence, the spindle speeds corresponding to the consecutive lobes should exhibit the same epsilon value (see Eq. (5)), and this affects the stability limit by generating the lobing effect. Thus, the spindle speeds along the stability diagram are selected such that each spindle speed corresponds to the minimum, i.e., absolute, stability limit of the corresponding lobe as shown in Fig. 5.

As shown in Fig. 5, the analytical absolute stability limit is significantly lower than $0.5 \mathrm{~mm}$. The experimental results are considered, and decreases in the spindle speed from $3750 \mathrm{r} / \mathrm{min}(140 \mathrm{~m} / \mathrm{min})$ to $2070 \mathrm{r} / \mathrm{min}(78 \mathrm{~m} / \mathrm{min})$ leads to an increase of stable cutting depth from $0.5 \mathrm{~mm}$ to $2 \mathrm{~mm}$. At a specific cutting experiment point of 2 $070 \mathrm{r} \cdot \mathrm{min}^{-1}$ and $1.75 \mathrm{~mm}$, the observed chatter frequency is $3113 \mathrm{~Hz}$. The experimental eigenvalue of the system is calculated as $\Lambda_{\exp }=-1.1314 \times 10^{6}+\mathrm{i} 2.0836 \times 10^{6}$ by Eq. (10). The experimental eigenvalue and the theoretical eigenvalue are equated to simultaneously solve the real and imaginary parts. Subsequently, the equations relating the total damping ratios to the experimental data are iteratively solved.

$$
\begin{aligned}
-\frac{1}{2 a_{0}}\left(a_{1} \pm \sqrt{a_{1}^{2}-4 a_{0}}\right) & =-1.1314 \times 10^{6}+\mathrm{i} 2.0836 \\
& \times 10^{6} .
\end{aligned}
$$

For this specific case, the solution of the above equation yields total damping ratios of $6.09 \%$ and $8.56 \%$ in the $x$ and $y$ directions, respectively. Finally, the average process damping coefficients are easily calculated by subtracting the structural damping ratio from the total damping ratio as

$c_{x}^{\mathrm{p}}=45.8 \mathrm{Ns} / \mathrm{m}, c_{x}^{\mathrm{p}}=72.9 \mathrm{Ns} / \mathrm{m}$. 
Table 1 Tool geometry and modal properties for the chatter tests

\begin{tabular}{llllll}
\hline Test & $\begin{array}{l}\text { Holder } \\
\text { length/mm }\end{array}$ & $\begin{array}{l}\text { Modal } \\
\text { stiffness } /\left(\mathrm{MN} \cdot \mathrm{m}^{-1}\right)\end{array}$ & $\begin{array}{l}\text { Modal } \\
\text { mass } / \mathrm{kg}\end{array}$ & $\begin{array}{l}\text { Natural } \\
\text { frequency/Hz }\end{array}$ & $\begin{array}{l}\text { Structural } \\
\text { damping ratio/\% }\end{array}$ \\
\hline 1 & 95 & 15.9 & 0.28 & 1195 & 1.49 \\
2 & 95 & 15.6 & 0.85 & 681 & 1.25 \\
\hline
\end{tabular}

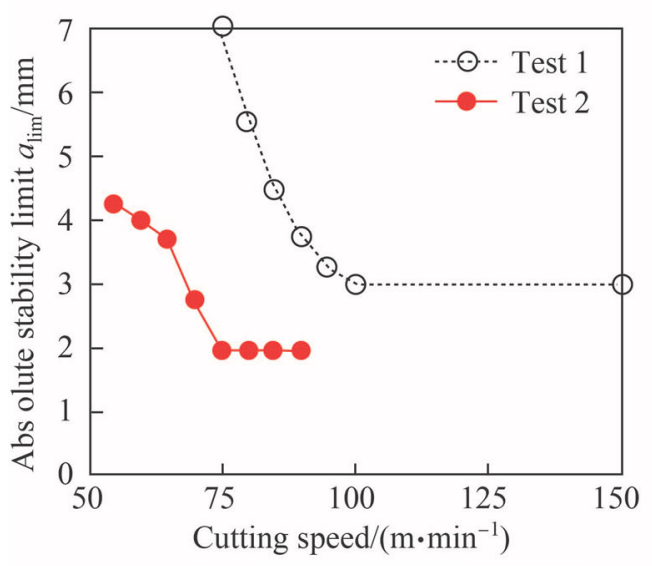

(a)

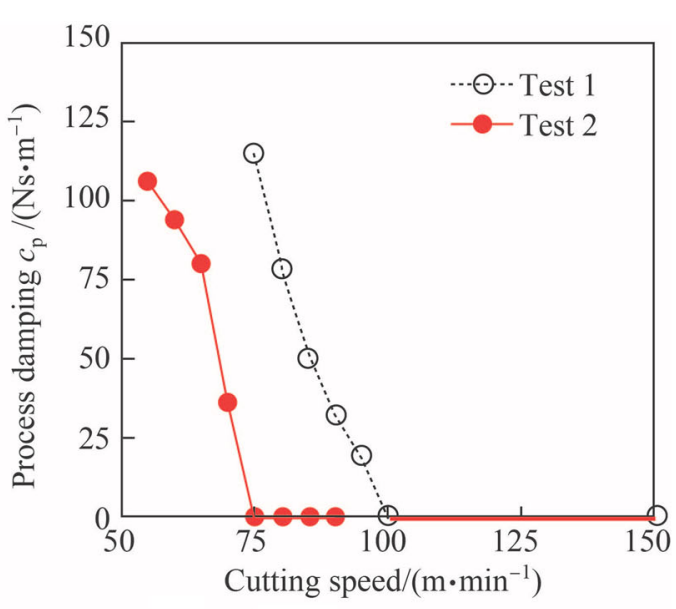

(b)

Fig. 4 Experimental results in orthogonal cutting. a Stability limits. b Process damping coefficient

Table 2 Cutting conditions and milling system for the end milling case [34]

\begin{tabular}{|c|c|c|c|c|c|c|c|c|c|c|c|}
\hline \multicolumn{6}{|c|}{ Cutting parameters } & \multicolumn{6}{|c|}{ Modal parameters } \\
\hline & & & & & & \multicolumn{2}{|c|}{$k /\left(\mathrm{N} \cdot \mu \mathrm{m}^{-1}\right)$} & \multicolumn{2}{|l|}{$\zeta / \%$} & \multicolumn{2}{|l|}{$f / \mathrm{Hz}$} \\
\hline Material & $K_{\mathrm{t}}, K_{\mathrm{r}} / \mathrm{MPa}$ & Tool diameter/mm & \# of flutes & Mode & Radial/\% & $x$ & $y$ & $x$ & $y$ & $x$ & $y$ \\
\hline AL7075 & 1050,300 & 12 & 4 & Down & 50 & 9.73 & 10.35 & 1.42 & 1.68 & 3110 & 3106 \\
\hline
\end{tabular}

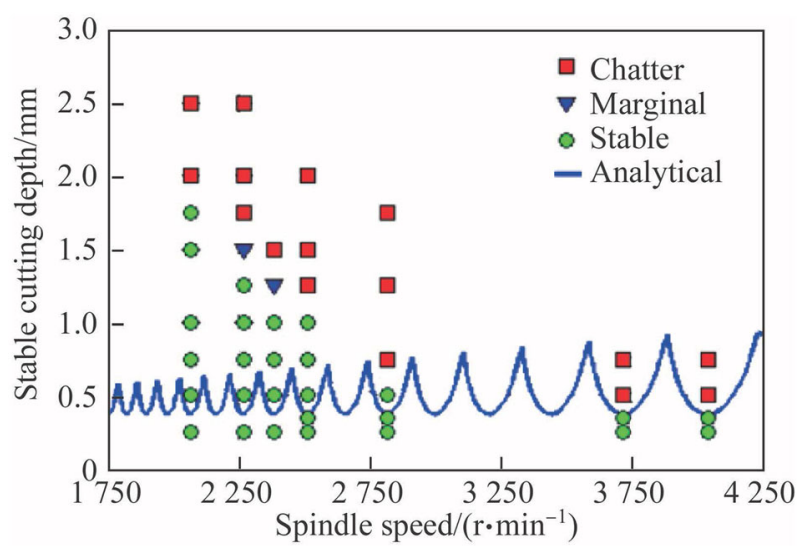

Fig. 5 Experimental stability limits for the example case

\section{Inverse stability solution at high cutting speeds}

In most stability analyses, the tool tip FRF is measured at the idle and even at the cold state of the spindle. However, at high rotational speeds and under operational conditions, the dynamics contributed by the bearings may change and lead to changes in the spindle dynamics when compared to that at the idle state. The aforementioned changes could be due to factors such as temperature increases, gyroscopic effects, and variation in the spindle preload with the spindle speed. The resulting variation may be due to the weighted combination of the aforementioned sources. Thus, experimental identification methods are required to accurately identify the variations since extant modeling efforts may lead to accuracy issues. In this section, the inverse stability solution approach is briefly explained, and a representative case study is presented. 

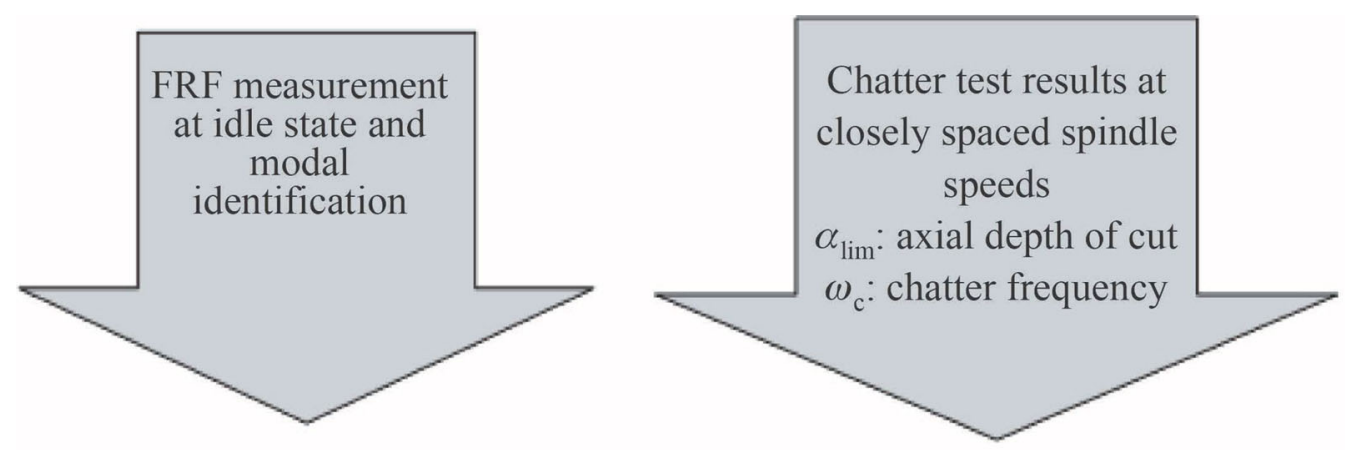

Set modal parameters of the dominant mode as unknown:

$$
\begin{aligned}
& \omega_{\mathrm{n}}: \xi \rightarrow x \text { direction } \\
& \omega_{\mathrm{n}}: \xi \rightarrow y \text { direction }
\end{aligned}
$$

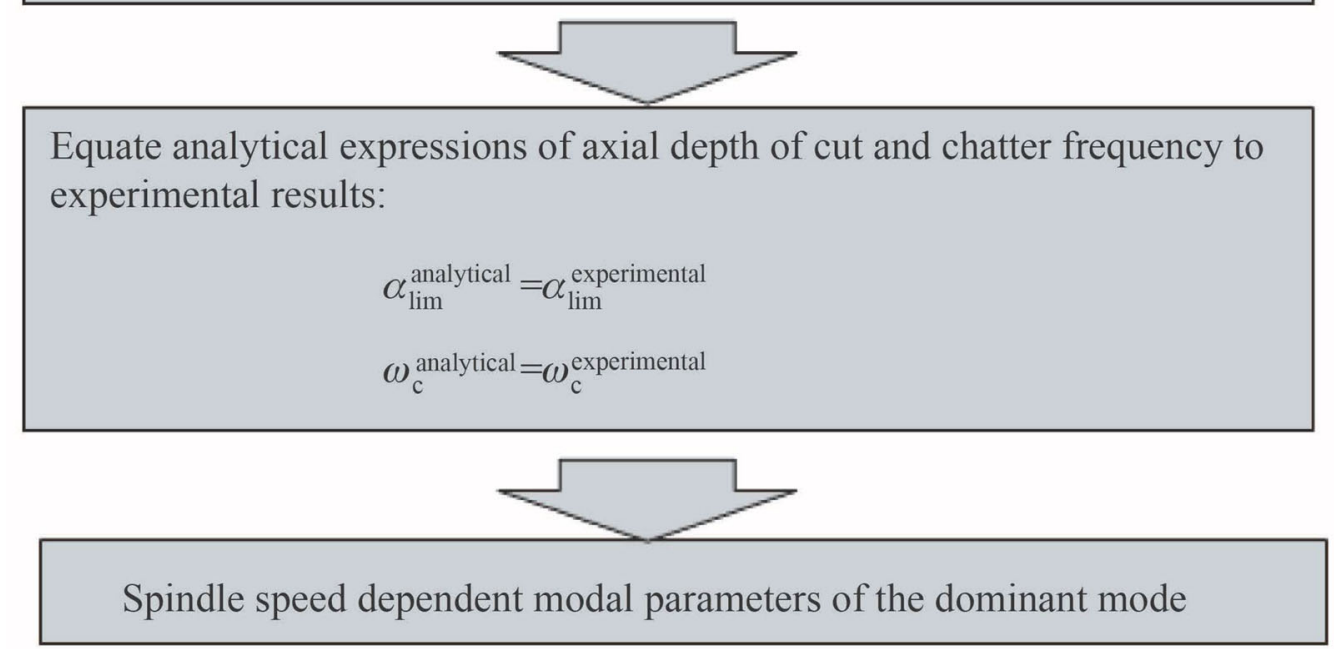

Fig. 6 Procedure of the tool point FRF identification

\subsection{Operational modal analysis for FRF identification}

In this study, the approach proposed by Ozsahin et al. [33] to identify the tool point FRF under operational conditions is considered as an inverse stability solution that addresses the uncertainties at high rotational speeds. In this method, at a given spindle speed, the stability limit and corresponding chatter frequency are experimentally obtained and then used in the inverse solution of the stability formulation (see Fig. 6). Analytical expressions for the chatter frequency and the axial depth of the cut are equated to the experimentally determined values.

Chatter can be caused by either the flexibility of the workpiece or the flexibility of the machining system. In the study, the workpiece is assumed rigid in which all the dynamic compliance is caused by the machine tool assembly. Thus, analytical expressions for the stability limit and chatter frequency contain parameters based on process dynamics of the machining operation and structural dynamics of the spindle-holder-tool assembly as given in Eq. (10). In process dynamics, with respect to a given radial immersion, the number of tooth, pitch angle, helix angle, and cutting force coefficients can be determined analytically or experimentally based on the workpiece material. However, in structural dynamics variations at the tool tip, it is difficult to measure or predict the FRF without speed dependent bearing and assembly dynamics information. Therefore, the modal parameters of the dominant mode in the tool point FRF can be treated as unknown parameters in analytical expressions for the axial stability limit and chatter frequency while maintaining the rest of the modes as corresponding to an idle state. Finally, an analytically obtained expression can be equated to the experimentally measured values, and the unknown modal parameters can be identified by solving nonlinear sets of 
equations. Additionally, it should be noted that a direct relation exists between the stiffness of the system and the natural frequency of the corresponding mode as given in Eq. (6), and thus only the natural frequency and damping ratio of the dominant mode are considered as unknowns. This is a valid approach since the modal mass is assumed as constant under operational conditions, and the variation in the stiffness is already reflected in the identified natural frequencies.

\subsection{Case study}

The method is demonstrated on a high-speed machining center by using a relatively rigid end mill with $25 \mathrm{~mm}$ diameter and 4 teeth. The stability diagram is generated by using the tool point FRF measured at the idle state. The results of the chatter tests performed at different speeds are given in Fig. 7 and Table 3. A high difference between the predicted and actual chatter frequencies indicates that the shift in the natural frequency corresponds to the dominant mode.

The natural frequency and damping ratio of the dominant mode that cause chatter can be identified as shown in Table 4 by using the inverse stability identification procedure. As shown in the identification results given in Table 4, the dominant mode exhibits a speed dependent behavior, and deviation in the natural frequency increases when the spindle speed increases. This is an expected result since it is known that stiffness values of the bearings decrease due to the gyroscopic moments and centrifugal forces. Therefore, the decrease in the bearing stiffness values results in a decrease in the natural frequencies.

In addition to identification results given in Table 4, the first tool point FRF is calculated by using the identified parameters for the $14000 \mathrm{r} / \mathrm{min}$ to quantify the accuracy of the identified mode. Subsequently, the stability diagram is calculated by using the idle FRF and updated FRF as shown in Fig. 8.

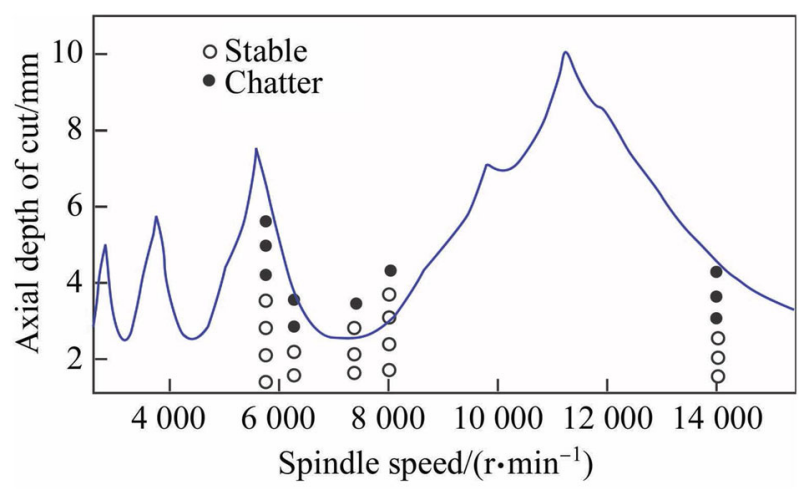

Fig. 7 Stability diagram predicted by using the idle state FRF and chatter test results [33]
As shown in Fig. 8, the use of the recalculated stability diagrams can predict the actual stability more accurately. Additionally, it is noted that the recalculated stability diagram shown in Fig. 8 is calculated by using the tool point FRF identified at $14000 \mathrm{r} / \mathrm{min}$, and thus it is valid only for the corresponding spindle speed. Therefore, to accurately predict the chatter stability at different spindle speeds, tool point FRFs should be identified at each considered spindle speed. Therefore, to obtain a stability diagram that is valid for a wide range of spindle speeds, tool point FRF should be separately identified at each spindle speed, and the obtained results should be separately combined.

The main contribution of the inverse stability solution for the identification of in-process tool point FRFs is that it eliminates the need for expensive measurement devices and complicated experimental setups. The approach provides a fast, easy, and reliable tool to identify tool point FRF under operational conditions.

\section{Conclusions}

Machining processes may demonstrate complicated dynamics in which stability analysis is of substantial importance in achieving improvements in part quality and process performance. Tool tip FRF is required for the analysis of dynamics and stability. However, in most cases, either at low cutting speeds or high rotational speeds, uncertainties may be introduced by either the process-cutting tool interaction or variations in the spindle dynamics. Although several studies focus on modeling the same, there is a paucity of research on inverse stability solution approaches that may be of immense help.

In this study, the approaches developed as an inverse solution of the stability problem to tackle the uncertainties in the dynamics of the machining processes are elaborated based on previous studies. The discussed identification methods are valid for a wide range of uncertainties that may be due to several sources. However, a low amount of uncertainty does not significantly affect the experimental stability boundaries, and thus the discussed methods inherently handle the significance issue. The process damping phenomenon and variation in spindle dynamics come into effect by classifying the machining processes into low cutting speed and high rotational speeds. In the study, the results indicate that the use of an inverse solution approaches works well in both conditions. At low cutting speeds, given the assumption that the rest of the parameters stay almost constant, the increase in the stability limit can be only attributed to process damping. Given this assumption, in orthogonal cutting, the process damping coefficient is directly related to the ratio of the experimental stability limits to the theoretical stability limits. 
Table 3 Predicted and actual values of the chatter frequency and axial depth of cut [33]

\begin{tabular}{lllll}
\hline Spindle speed $/\left(\mathrm{r} \cdot \mathrm{min}^{-1}\right)$ & $\begin{array}{l}\text { Predicted } \\
\text { stability limit } \\
a_{\mathrm{lim}} / \mathrm{mm}\end{array}$ & $\begin{array}{l}\text { Predicted } \\
\text { chatter frequency } \\
f_{\mathrm{c}} / \mathrm{Hz}\end{array}$ & $\begin{array}{l}\text { Actual } \\
\text { stability limit } \\
a_{\lim } / \mathrm{mm}\end{array}$ & $\begin{array}{l}\text { Actual } \\
\text { chatter frequency } \\
f_{\mathrm{c}} / \mathrm{Hz}\end{array}$ \\
\hline 6300 & 3.60 & 732.0 & 2.6 & 697 \\
7500 & 2.42 & 764.4 & 3.1 & 728 \\
14000 & 4.45 & 722.8 & 3.0 & 616 \\
\hline
\end{tabular}

Table 4 Identified modal parameters in the $y$ direction at various spindle speeds [33]

\begin{tabular}{lllll}
\hline & $\begin{array}{l}\text { Spindle speed } \\
/\left(\mathrm{rmin}^{-1}\right)\end{array}$ & $\begin{array}{l}\text { Spindle speed } \\
6200-6300 \mathrm{r} / \mathrm{min}\end{array}$ & $\begin{array}{l}\text { Spindle speed } \\
7400-7500 \mathrm{r} / \mathrm{min}\end{array}$ & $\begin{array}{l}\text { Spindle speed } \\
13900-14000 \mathrm{r} / \mathrm{min}\end{array}$ \\
\hline $\begin{array}{l}\text { Natural frequency/Hz } \\
\text { Damping/\% }\end{array}$ & 744 & 672 & 642 & 611 \\
\hline
\end{tabular}

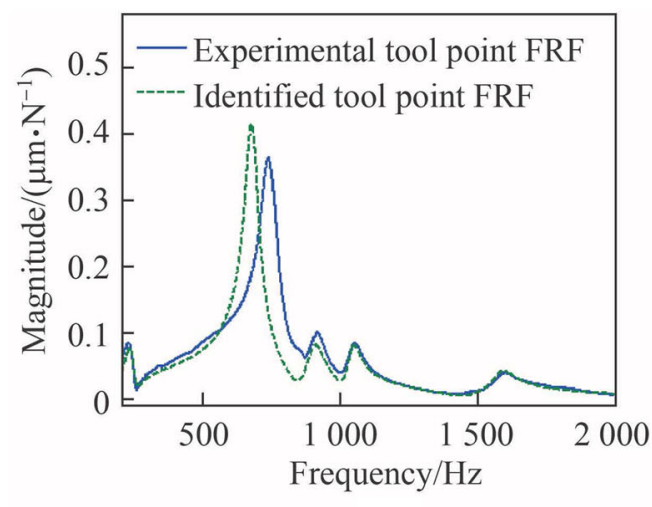

(a)

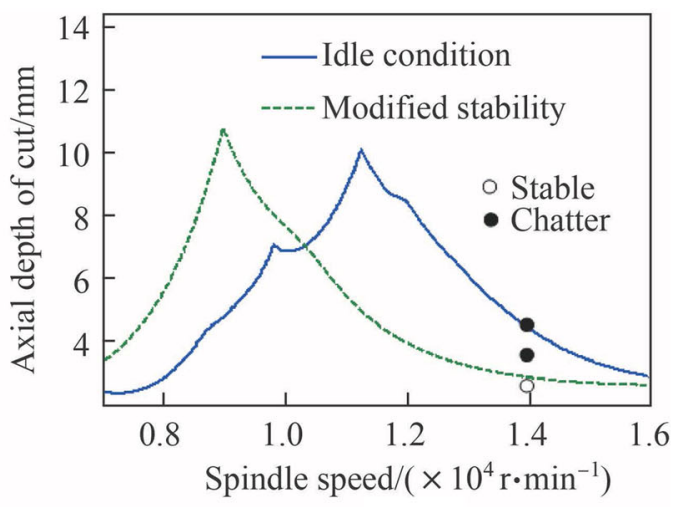

(b)

Fig. 8 Variation in the tool tip FRF and the corresponding stability diagram a tool point FRF at idle and at $14000 \mathrm{r} / \mathrm{min} \mathbf{b}$ stability diagrams at idle and $14000 \mathrm{r} / \mathrm{min}[33]$

Similarly, in milling, the average process damping coefficients are calculated by using an iterative inverse solution. At high rotational speeds, the variation in the spindle dynamics causes the tool tip FRF to change, and this could be due to several factors. In the study, the findings reveal that once the experimental stability limit and the corresponding chatter frequency are identified, the inverse solution of the stability equations leads to the accurate prediction of the in-process tool tip FRF as opposed to that measured at the idle state.

Although the study does not propose a new model, the aim involves discussing the inverse stability solutions for different cases in a comprehensive manner based on extant studies. In this aspect, turning and milling case studies are discussed to elaborate the use of an inverse stability solution for process damping identification while a milling case is considered to discuss the use of inverse stability solution for milling at high rotational speeds.
Acknowledgements The authors acknowledge the support of Turkish National Science Foundation (Grant No. 108M340).

\section{References}

1. Taylor FW (1907) On the art of cutting metals. American Society of Mechanical Engineers, New York

2. Altintas Y, Budak E (1995) Analytical prediction of stability lobes in milling. Ann CIRP 44(1):357-362

3. Insperger T, Stepan G (2002) Semi-discretization method for delayed systems. Int J Numer Methods Eng 55(5):503-518

4. Tlusty J, Polacek M (1963) The stability of machine tools against self-excited vibrations in machining. Int Res Prod Eng 465-474

5. Das MK, Tobias SA (1967) The relation between the static and the dynamic cutting of metals. Int J Mach Tool Des Res 763:89

6. Koenigsberger F, Tlusty J (1967) Machine tool structures-Vol. I: stability against chatter. Pergamon Press, Oxford

7. Opitz H, Bernardi F (1970) Investigation and calculation of the chatter behavior of lathes and milling machines. Ann CIRP 18:335-343 
8. Sridhar R, Hohn RE, Long GW (1968) A stability algorithm for the general milling process: contribution to machine tool chatter research 7. J Eng Ind 90(2):330-334

9. Minis I, Yanushevsky T, Tembo R et al (1990) Analysis of linear and nonlinear chatter in milling. Ann CIRP 39:459-462

10. Tlusty J (1978) Analysis of the state of research in cutting dynamics. Ann CIRP 27(2):583-589

11. Sisson TR, Kegg RL (1969) An explanation of low-speed chatter effects. ASME J Eng Ind 91:558-951

12. Wu DW (1984) A new approach of formulating the transfer function for dynamic cutting processes. J Eng Ind 111:37-47

13. Budak E, Tunc LT (2009) A new method for identification and modeling of process damping in machining. J Manuf Sci Eng 131(5):051019

14. Rivin E (1999) Stiffness and damping in mechanical design. Marcel Dekker Inc., New York

15. Stone BJ (1982) The state of the art in the measurement of the stiffness and damping of rolling element bearings. CIRP Ann Manuf Technol 31:529-538

16. Harris TA (2001) Rolling bearing analysis, 4th edn. Wiley, New York

17. Lee CW (1993) Vibration analysis of rotors. Kluwer, Dordrecht

18. Friswell MI, Penny JET, Garvey SD et al (2010) Dynamics of rotating machines. Cambridge University Press, Cambridge

19. Jiang JS, Zheng S (2010) A modeling approach for analysis and improvement of spindle-drawbar-bearing assembly dynamics. Int J Mach Tools Manuf 50(1):131-142

20. Kruth JP, Liu AMM, Vanherck P et al (2002) A strategy for selection of optimal cutting parameter in high-speed milling to avoid chatter vibration. Int J Prod Eng Comput 4(5):35-42

21. Kilic ZM, Iglesias A, Munoa J et al (2010) Investigation of tool wear on the stability of milling process using an inverse method. In: CIRP 2nd international conference on process machine interactions, Vancouver, Canada

22. Suzuki N, Kurata Y, Kato T et al (2012) Identification of transfer function by inverse analysis of self-excited chatter vibration in milling operations. Precis Eng 36(4):568-575

23. Cao Y, Altintas Y (2004) A general method for the modeling of spindle-bearing systems. J Mech Des 126(6):557-566
24. Altintas Y, Cao Y (2005) Virtual design and optimization of machine tool spindles. CIRP Ann Manuf Technol 54(1):379-382

25. Lin CW, Tu JF, Kamman J (2003) An integrated thermo-mechanical-dynamic model to characterize motorized machine tool spindles during very high-speed rotation. Int J Mach Tools Manuf 43(10):1035-1050

26. Tatar K, Gren P (2007) Measurement of milling tool vibrations during cutting using laser vibrometry. Int J Mach Tools Manuf 48:380-387

27. Rantatalo M, Aidanpaa JO, Göransson B et al (2007) Milling machine spindle analysis using FEM and non-contact spindle excitation and response measurement. Int J Mach Tools Manuf 47:1034-1045

28. Zaghbani I, Songmene V (2009) Estimation of machine-tool dynamic parameters during machining operation through operational modal analysis. Int J Mach Tools Manuf 49:947-957

29. Opitz H, Weck MC (1970) Determination of the transfer function by means of spectral density measurements and its application to dynamic investigation of machine tools under machining conditions. In: Proceedings of the 10th international MTDR conference, University of Manchester Institute of Science and Technology, Manchester

30. Minis IE, Magrab EB, Pandelidis IO (1990) Improved methods for the prediction of chatter in turning Part 1: determination of structural response parameters. Trans ASME 112:12-20

31. Ozsahin O, Budak E, Ozguven HN (2011) Investigating dynamics of machine tool spindles under operational conditions. Adv Mater Res 223:610-621

32. Bediz B, Kumar U, Schmitz TL et al (2012) Modeling and experimentation for three-dimensional dynamics of endmills. Int J Mach Tools Manuf 53(1):39-50

33. Ozsahin O, Budak E, Ozguven HN (2015) In-process tool point FRF identification under operational conditions using inverse stability solution. Int J Mach Tools Manuf 89:64-73

34. Tunç LT, Budak E (2013) Identification and modeling of process damping in milling. Trans ASME J Manuf Sci Eng 135(2):021001 